\title{
Some applications of CHEVIE to the theory of algebraic groups
}

\section{MEINOLF GECK}

\section{ABSTRACT.}

The computer algebra system CHEVIE is designed to facilitate computations with various combinatorial structures arising in Lie theory, like finite Coxeter groups and Hecke algebras. We discuss some recent examples where CHEVIE has been helpful in the theory of algebraic groups, in questions related to unipotent classes, the Springer correspondence and Lusztig families.

\section{REFERENCES}

[1] Achar, P. and Aubert, A.-M., Supports unipotents de faisceaux caractères, J. Inst. Math. Jussieu 6 (2007), 173-207

[2] Adams J., et al., Atlas of Lie groups and representations, http: / /www . liegroups .org/

[3] Bellamy, G., On singular Calogero-Moser spaces, Bull. Lond. Math. Soc. 41 (2009), 315-326

[4] Beynon, W. M. and Spaltenstein, N., Green functions of finite Chevalley groups of type $E_{n}(n=6,7,8)$, J. Algebra 88 (1984), $584-614$

[5] Bonnafé, C., Semicontinuity properties of Kazhdan-Lusztig cells, preprint (2008), New Zealand J. Math. (to appear)

[6] Broué, M. and Malle, G., Zyklotomische Hecke-Algebren, in Représentations unipotentes génériques et blocs des groupes réductifs finis, Astérisque No. 212 (1993), 119-189

[7] Broué, M., Malle, G. and Michel, J., Towards Spetses I, dedicated to the memory of Claude Chevalley, Transform. Groups 4 (1999), 157-218

[8] Carter, R. W., Finite groups of Lie type: Conjugacy classes and complex characters, Wiley, New York, 1985; reprinted 1993 as Wiley Classics Library Edition

[9] Char, B. W., Geddes, K. O., Gonnet, G. H., Leong, B. L., Monagan, M. B., Watt, S. M., Maple V, Language Reference Manual, Springer, New York, 1991

[10] Casselman, B., Verifying Kottwitz' conjecture by computer, Represent. Theory 4 (2000), 32-45 (electronic)

[11] Curtis, C. W. and Reiner, I., Methods of representation theory Vol. I and II, Wiley, New York, 1981 and 1987

[12] DuCloux, F., Positivity results for the Hecke algebras of non-crystallographic finite Coxeter group, J. Algebra 303 (2006), 731-741; section "Computational Algebra"

[13] Enomoto, H., The characters of the finite symplectic group $\operatorname{Sp}(4, q), q=2^{f}$, Osaka J. Math. 9 (1972), 75-94

[14] Geck, M., On the average values of the irreducible characters of finite groups of Lie type on geometric unipotent classes, Doc. Math. 1 (1996), 293-317 (electronic)

[15] Geck, M., Constructible characters, leading coefficients and left cells for finite Coxeter groups with unequal parameters, Represent. Theory 6 (2002), 1-30 (electronic)

[16] Geck, M., An introduction to algebraic geometry and algebraic groups, Oxford Graduate Texts in Mathematics 10, Oxford University Press, New York, 2003

[17] Geck, M., Computing Kazhdan-Lusztig cells for unequal parameters, J. Algebra 281 (2004), 342-365; section "Computational Algebra"

[18] Geck, M., Hecke algebras of finite type are cellular, Invent. Math. 169 (2007), 501-517

[19] Geck, M., Leading coefficients and cellular bases of Hecke algebras, Proc. Edinburgh Math. Soc. 52 (2009), 653-677

[20] Geck, M., On the Kazhdan-Lusztig order on cells and families, Comment. Math. Helv. (to appear)

[21] Geck, M., Hiß, G., Lübeck, F., Malle, G. and Pfeiffer, G., CHEVIE-A system for computing and processing generic character tables for finite groups of Lie type, Weyl groups and Hecke algebras, Appl. Algebra Engrg. Comm. Comput. 7 (1996), 175-210

[22] Geck, M. and Iancu, L., On the Kazhdan-Lusztig order on families in type $B_{n}$, preprint (2010) (in preparation)

[23] Geck, M. and Malle, G., On special pieces in the unipotent variety, Experimental Math. 8 (1999), 281-290

[24] Geck, M. and Malle, G., On the existence of a unipotent support for the irreducible characters of finite groups of Lie type, Trans. Amer. Math. Soc. 52 (2000), 429-456

[25] Geck, M. and Michel, J., “Good" elements in finite Coxeter groups and representations of Iwahori-Hecke algebras, Proc. London Math. Soc. 74 (1997), 275-305

[26] Geck, M. and Pfeiffer, G., On the irreducible characters of Hecke algebras, Advances in Math. 102 (1993), 79-94

[27] Geck, M. and Pfeiffer, G., Characters of finite Coxeter groups and Iwahori-Hecke algebras, London Math. Soc. Monographs, New Series 21, Oxford University Press, New York, 2000

[28] Gomi, Y., The Markov traces and the Fourier transforms, J. Algebra 303 (2006), 566-591

[29] Guilhot, J., Kazhdan-Lusztig cells in the affine Weyl groups of rank 2, Int. Math. Res. Notices, vol. 2010, Article ID rnp242, 41 pages; doi:10.1093/imrn/rnp243

[30] He, X., On the affineness of Deligne-Lusztig varieties, J. Algebra 320 (2008), 1207-1219

[31] Himstedt, F. and Huang, S.-C., Character tables of the maximal parabolic subgroups of the Ree groups ${ }^{2} F_{4}\left(q^{2}\right)$, LMS J. Comput. Math. 13 (2010), 90-110

[32] Kazhdan, D. A. and Lusztig, G., Representations of Coxeter groups and Hecke algebras, Invent. Math. 53 (1979), 165-184

[33] van Leeuwen, M. A. A., Cohen, A. M. and Lisser, B., LiE, a package for Lie group computations, http://www-math.univ-poitiers.fr/ $\sim$ maavl/LiE/

[34] Lübeck, F., Tables of Green functions for exceptional groups, http://www/math.rwth-aachen. de/ Frank. Luebeck/chev/Green/

[35] Lusztig, G., On the finiteness of the number of unipotent classes, Invent. Math. 34 (1976), 201-213

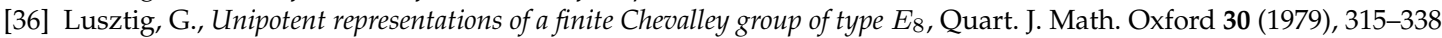

[37] Lusztig, G., A class of irreducible representations of a finite Weyl group, Indag. Math. 41 (1979), 323-335

[38] Lusztig, G., On the unipotent characters of the exceptional groups over finite fields, Invent. Math. 60 (1980), 173-192

Received: 06.08.2010; In revised form: 20.12.2010; Accepted: 23.02.2011

2000 Mathematics Subject Classification. 20C40, 20 G40.

Key words and phrases. Computer algebra, algebraic groups, Coxeter groups. 
[39] Lusztig, G., A class of irreducible representations of a finite Weyl group II, Indag. Math. 44 (1982), 219-226

[40] Lusztig, G., Left cells in Weyl groups, in Lie group representations, I (College Park, Md., 1982/1983), Lecture Notes in Math., 1024, Springer, Berlin, 1983, 99-111

[41] Lusztig, G., Characters of reductive groups over a finite field, Annals Math. Studies, vol. 107, Princeton University Press, 1984

[42] Lusztig, G., Intersection cohomology complexes on a reductive group, Invent. Math. 75, 205-272 (1984)

[43] Lusztig, G., Character sheaves V, Adv. Math. 61 (1986), 103-155

[44] Lusztig, G., Green functions and character sheaves, Ann. Math. 131 (1990), 355-408

[45] Lusztig, G., Exotic Fourier transform, with an appendix by Gunter Malle, Duke Math. J. 73 (1994), 227-241, 243-248

[46] Lusztig, G., Notes on unipotent classes, Asian J. Math. 1 (1997), 194-207

[47] Lusztig, G., Hecke algebras with unequal parameters, CRM Monographs Ser. 18, Amer. Math. Soc., Providence, RI, 2003

[48] Lusztig, G., Homomorphisms of the alternating group $\mathcal{A}_{5}$ into reductive groups, J. Algebra 260 (2003), 298-322

[49] Lusztig, G., Unipotent classes and special Weyl group representations, J. Algebra 321 (2009), 3418-3440

[50] Lusztig, G., On some partitions of a flag manifold, peprint (2009), available at arXiv:0906.1505

[51] Lusztig, G., From conjugacy classes in the Weyl group to unipotent classes, Represent. Theory (to appear)

[52] Lusztig, G., Elliptic elements in a Weyl group: a homogeneity property, preprint (2010), available at arXiv: 1007.5040

[53] Lusztig, G. and Spaltenstein, N., On the generalized Springer correspondence for classical groups, in Algebraic groups and related topics (Kyoto/Nagoya, 1983), Adv. Stud. Pure Math., 6, North-Holland, Amsterdam, 1985, 289-316

[54] Malle, G., Appendix: An exotic Fourier transform for $H_{4}$, Duke J. Math. 73 (1994), 243-248

[55] Michel, J., Homepage of the development version of the GAP part of CHEVIE, http://www.institut.math.jussieu.fr/ jmichel/ chevie/chevie.html

[56] Reeder, M., Formal degrees and L-packets of unipotent discrete series representations of exceptional p-adic groups, with an appendix by Frank Lübeck, J. Reine Angew. Math. 520 (2000), 37-93

[57] Schönert, M. et al., GAP - Groups, Algorithms, and Programming, Lehrstuhl D für Mathematik, RWTH Aachen, Germany, fourth ed., 1994

[58] Serre, D., Matrices. Theory and applications, Translated from the 2001 French original, Graduate Texts in Mathematics, vol. 216, Springer-Verlag, New York, 2002

[59] Shoji, T., On the Green polynomials of classical groups, Invent. Math. 74 (1983), 239-267

[60] Shoji, T., Green functions of reductive groups over a finite field, in The Arcata Conference on Representations of Finite Groups (Arcata, Calif., 1986), Proc. Sympos. Pure Math., 47, Part 1, Amer. Math. Soc., Providence, RI, 1987, 289-302

[61] Shoji, T., Character sheaves and almost characters of reductive groups, II, Advances in Math. 111 (1995), 314-354

[62] Spaltenstein, N., Classes unipotentes et sous-groupes de Borel, Lecture Notes in Math., 946, Springer, 1982

[63] Spaltenstein, N., A property of special representations of Weyl groups, J. Reine Angew. Math. 343 (1983), 212-220

[64] Spaltenstein, N., On the generalized Springer correspondence for exceptional groups, in Algebraic groups and related topics (Kyoto/Nagoya, 1983), Adv. Stud. Pure Math., 6, North-Holland, Amsterdam, 1985, 317-338

[65] Springer, T. A., Trigonometric sums, Green functions of finite groups and representations of Weyl groups, Invent. Math. 36 (1976), 173-207

[66] Srinivasan, B., The characters of the finite symplectic group $\operatorname{Sp}(4, q)$, Trans. Amer. Math. Soc. 131 (1968), 488-525

INSTITUTE OF MATHEMATICS

UNIVERSITY OF ABERDEEN

ABERDEEN AB24 3UE, UK

E-mail address: m. geck@abdn. ac.uk 